\title{
COMMENTARY
}

\section{Effect of Whitening Dentifrices on the Surface Roughness of Commercial Composites}

\section{GISELE F. NEIVA, DDS, MS*}

The authors of this article addressed a very current topic that deals with the surface characteristics of composite resin restorations when subjected to the inevitable daily exposure to toothbrush/toothpaste abrasion. This study was carefully designed and it was carried out with similar concern. It demonstrated that whitening toothpastes, generally more abrasive than regular dentifrices, cause greater surface changes to composite restorations. The wear pattern was cumulative and was shown to be progressively worse with the increased exposure time. However, contrary to what was expected, samples of a microhybrid composite resulted in rougher surfaces when compared with samples of a posterior hybrid composite after simulated 24 months of brushing. This goes against the evidence in the literature as similar studies have demonstrated that posterior hybrid composites are generally attributed to rougher surfaces.

Two different composites were used in the study: a universal microhybrid (Filtek Z250 by 3 M/ESPE, St. Paul, MN, USA) and a posterior hybrid (Rok by SDI, Australia). Considering that wear of composites is know to be dependent on filler particle shape, size, and distribution, it is prudent to further discuss the differences between these formulations. Filtek Z250 has zirconium and silica as fillers with round-shaped particles ranging from 0.01 to $3.5 \mu \mathrm{m}$ in diameter whereas the strontium-aluminum silicate irregularly shaped particles in Rok range between $40 \mathrm{~nm}$ and $2.5 \mu \mathrm{m}$. The literature shows compelling evidence of reduced composite wear associated with smaller particle sizes. Even though Filtek Z250 has smaller mean particle sizes $(0.6 \mu \mathrm{m}$ versus $1.2 \mu \mathrm{m}$ in Rok) the range includes particles that can be potentially five to six times larger than the reported average. Therefore, part of the variation observed could still be attributed to filler size and distribution in the formulation. Also, round particles are not "self-retentive" and may be more easily dislodged than irregular ones as a result of the matrix wear and dissolution (as demonstrated by the authors in Figures $4 \mathrm{C}$ and D). It is reasonable to assume that the Surface Roughness Tester used in the study captured the voids left by the dislodged round particles in Filtek Z250 samples in addition to the generalized roughness of the abraded composite matrix, which potentially accounted for the overestimated effect reported in the study. Nonetheless, the discussion was very enlightening and elucidated some of the other sources of variation apart from filler characteristics, namely, relative wear resistance of filler matrix, degree of resin conversion, and quality of the filler/matrix interface.

It would have been helpful if the authors could have provided more information about the abrasiveness of the dentifrices selected. Since 1998, acceptable RDA values (Radioactive Dentin Abrasion) have been published in the DIN EN ISO | 1609. The American Dental Association has since adopted the procedure for measuring the Relative Dentin Abrasion Index of dentifrices. Values above 150 RDA have been regarded as harmful and dentifrices that exceed this limit should be used with caution. It is uncertain whether any of the dentifrices used in this study exceed this threshold. However, it is reasonable to assume that highly abrasive dentifrices could have accounted for overestimated effects. Regardless of this minor criticism, I would like to congratulate the authors for their great work and for raising awareness about misuse of whitening dentifrices and their consequent deleterious effect on the surface of composite restorations.

*Clinical Associate Professor, Department of Cariology, Restorative Sciences \& Endodontics, The University of Michigan School of Dentistry, Ann Arbor, MI, USA

This commentary is accompanied by article, "Effect of Whitening Dentifrices on the Surface Roughness of Commercial Composites" Guilherme Machado Barbieri, DDS, MS, PhD, Eduardo Gonçalves Mota, DDS, MS, PhD, Sinval Adalberto Rodrigues-Junior, DDS, MS, PhD, Luiz Henrique Burnett Jr, DDS, MS, PhD, DOI I0.1 I I I/j. $1708-8240.2011 .00426 . x$. 